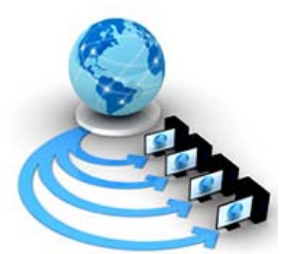

International Journal of Advanced Research in Computer Science

RESEARCH PAPER

\author{
Available Online at www.ijarcs.info
}

\title{
REAL TIME GESTURE RECOGNITION BASED EQUIPMENT AND MEDIA CONTROL OVER INTERNET PROTOCOL
}

\author{
Shiv Shanker Garg \\ Computer Science \& Engineering \\ Apex Institute of Engineering \& Technology \\ Jaipur, India
}

\author{
Manish Kumar \\ Computer Science \& Engineering \\ Apex Institute of Engineering \& Technology \\ Jaipur, India
}

\author{
Brij Kishore \\ Computer Science \& Engineering \\ Apex Institute of Engineering \& Technology \\ Jaipur, India
}

\begin{abstract}
Signal is a standout amongst the most characteristic and expressive methods for interchanges amongst human and PC in a genuine framework. We normally utilize different motions to express our own particular aims in regular day to day existence. Hand signal is one of the critical strategies for non-verbal correspondence for individuals. Hand motion acknowledgment based man-machine interface is being produced energetically as of late. This paper gives an ongoing vision based technique for perceiving the hand signals utilizing MATLAB. It additionally gives the working subtle elements of acknowledgment handle utilizing Edge identification and Skin recognition calculations. Signals perceived are then utilized for media control, for example, Photo show, Audio Video Streams Playback, basic media control elements, for example, Play/Pause, Forward, Reverse, Stop Etc. Likewise our proposed framework permits to catch and arrange sound or video or joint catching utilizing hand motion flags as it were. The equipment interface innovation utilized enables us to control home apparatuses framed in a remote system by means of hand motion developments just, this opens another pleothera of roads progressively vision based home mechanization frameworks.
\end{abstract}

Keywords: Feature extraction; Gesture recognition; Image segmentation; Image processing ; Neural network.

\section{INTRODUCTION}

There has been an incredible accentuation of late on HumanPC Interaction (HCI) research to make simple to-utilize interfaces by specifically utilizing normal correspondence and control abilities of people. Among various human body parts, the hand is the best broadly useful collaboration device, because of its mastery. The word motion is utilized for a wide range of marvels including human development, particularly of the hands and arms, just some of these are intuitive or open. The primary method for collaboration with PC utilizing hand signal was first proposed by Myron W. Krueger in 1970. Receiving hand signal as an interface in HCI will permit the organization of an extensive variety of applications with no physical contact with the processing conditions.[1] These days, dominant part of the HCI depends on mechanical gadgets, for example, console, mouse, joystick or amusement cushion, however a developing enthusiasm for a class of techniques based on computational vision has been risen because of capacity to perceive human motions normally. The principle motivation behind motion acknowledgment is to distinguish a specific human motion and pass on data to the PC. General point is to make the PC comprehend human motions, to control remotely through hand acts a wide assortment of gadgets. The programmed vision-based acknowledgment of hand motion for communication through signing and for control of electronic gadgets, as advanced TV, play stations was consider as a hot investigate point as of late. Be that as it may, the general issues of these works ascend because of many issues, for example, the complex foundations, the skin shading and the idea of static and dynamic hand signals. Hand signals acknowledgment for
TV control is proposed by. In this framework, just a single signal is utilized to control TV by moving client hand. On the show, a hand symbol shows up which takes after the client's hand. The standardized connection was utilized to examine the client's hand. A PC interface in view of hand signal acknowledgment for controlling applications like media player utilizing PC vision methods, is proposed by. In this calculation, a main part investigation for signal pictures utilized for hand highlight extraction, while, the distinguishing the hand movement depended on utilizing Lucas Kanade Pyramidical optical stream calculation. By utilizing K-Nearest Neighbor calculation, the acknowledgment of the motion is accomplished. A survey of the history of motion controlled UI, and its application is exhibited in. The proposed system perceive an arrangement of four particular hand signals, specifically: Play, pause \& play, notebook, image. Our approach bases on four stages, Image obtaining, Hand division, Features extraction, and Arrangement or acknowledgment. A counterfeit neural system (ANN) has been used as a motion classifier. The commitments of this paper are as per the following: $[1,2,3]$

1. We propose full of feeling method for hand signal acknowledgment to control media player utilizing the properties of hand picture locales by following the locale limit.

2. We tried and assess the effectiveness of our proposed calculation progressively to four particular hand signals, in particular: Play, Pause, Notebook, image . 


\section{Gesture Recognition System}

In following fig 1 the vast majority of the scientists ordered motion acknowledgment framework into for the most part three stages subsequent to procuring the info picture from camera, videos or even information glove instrumented gadget. These means are: Extraction strategy, Highlight extraction, and Classification or acknowledgment as represented in figure1. Picture catch stage is to obtain a picture, or an arrangement of pictures, which is then prepared in the following stages. The catching is for the most part done utilizing a solitary camera with a frontal perspective of the individual's hand, which plays out the signals. The essential point of this stage is to ideally set up the picture acquired from past stage keeping in mind the end goal to remove the highlights in next stage. How an ideal outcome looks like depends mostly on the subsequent stage. The point of this stage is to find and concentrate highlights that can be utilized to decide the significance of a given motion.

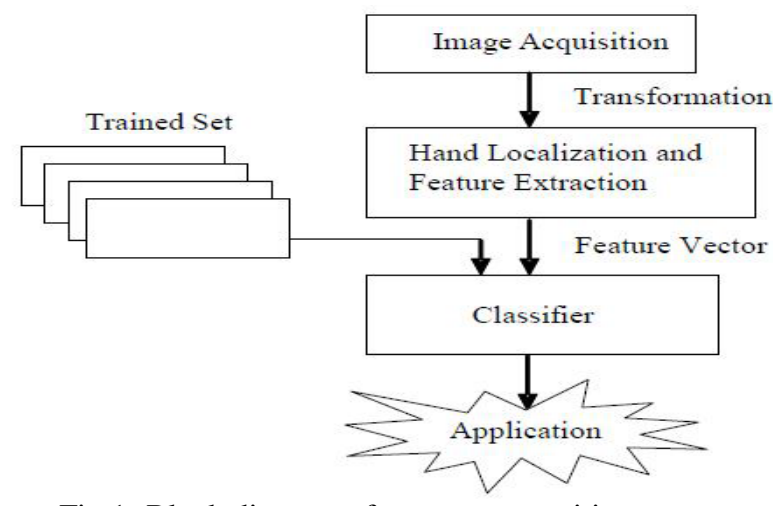

Fig.1: Block diagram of gesture recognition system

This stage extricates highlights for arrangement. The arrangement speaks to the undertaking of appointing an element or set of components to some predefined classes with a specific end goal to perceive the hand motion. In earlier years a few characterization techniques have been proposed and effectively tried in various acknowledgment frameworks. All in all, a class is characterized as an arrangement of reference highlights that were acquired amid the preparation period of the framework utilizing an arrangement of preparing pictures. Consequently the grouping for the most part comprises of finding the best coordinating reference highlights for the components removed in the past stage.[3,4]

\section{LITURATURE REVIEW}

Motion is a standout amongst the most clear and emotional method for correspondences amongst human and PC. Consequently, there has been a developing enthusiasm to make simple to-utilize interfaces by straightforwardly using the regular correspondence and administration aptitudes of people. This paper displays a hand signal interface for controlling media player utilizing neural system. The proposed calculation perceives an arrangement of four particular hand signals, to be specific: Play, Stop, Forward, and Reverse. Our calculation depends on four stages, Image securing, Hand division, Features extraction, and Classification. An edge from the webcam camera is caught, and afterward skin identification is utilized to fragment skin districts from foundation pixels. Another picture is made containing hand limit. Hand shape highlights extraction, are used to portray the hand motion. A simulated neural system has been used as a motion classifier, also. 120 signal pictures have been utilized for preparing. The acquired normal grouping rate is $95 \%$. The proposed calculation builds up an elective info gadget to control the media player, and furthermore offers diverse motion orders and can be valuable progressively applications. Examinations with other hand motion acknowledgment frameworks have uncovered that our framework demonstrates better execution in wording exactness. A PC vision calculation is recommended that perceives the four hand signals in particular: Play, Stop, Forward, and Reverse, for controlling media player utilizing neural system. An edge from the webcam camera was caught, at that point skin division utilizing $\mathrm{LAB}$ shading space was utilized to fragment skin districts from foundation pixels. Another picture was made containing hand limit of the client's hand. A curved structure also, corner location are utilized to depict the shape components of hand motion. A managed back-engendering multi-layer feed forward neural system was utilized for order of client's hand motions also. The arrangement was made, without the requirement for utilizing any unique apparatuses, for example, gloves or marker. In any case, the proposed calculation performed well in characterization the four clients' hand motion summon, with some characterization blunders. The normal characterization rate of $95 \%$ was gotten for the testing sets. As future work, we plan to utilize hand motion characterization calculation for controlling the TV, and portable applications. [5]

Signals are an effective methods for correspondence among people. Motion acknowledgment is the way toward perceiving and translating a stream consistent successive motion from the given arrangement of information. Essential Concern close by signal acknowledgment is that it could be workable for clients to speak with mechanized hardware without requirement for outside control gadgets. Another favorable position is that the client can convey from a separation, as well as need have no physical contact with the PC. As per overview just about $40 \%$ of all signals are finished with one hand and various hands at $20 \%$ and the rest are conveyed between the other body parts. In this technique uncovered hand motions are perceiving utilizing dynamic vision sensor (DVS) camera. DVS is not quite the same as ordinary cameras. DVS cameras just react to pixels with transient luminance contrasts, which can extraordinarily diminish the computational cost of contrasting back to back casings with track moving article. This technique endeavors to characterize three distinctive hand signals made by a client which is called as Rock, Paper, Scissors. This is novel strategy identify the point where the client conveys a toss, to extricate hand locales and to separate valuable components for characterization. Proposed framework proposes a strategy to characterize uncovered hand motion utilizing dynamic vision sensor (DVS) camera. This framework concentrated on ordering three distinct motions (shake, paper, and scissors) and utilizing those three motions mouse free interface can be outlined. In proposed framework there are five phases to perceive distinctive hand signals. To perceive a toss, the point where the player conveys a toss ought to be identified first; called as point as conveyance point. Once the conveyance point is distinguished, the framework can order distinctive tosses utilizing just a solitary stance caught at the conveyance point. After conveyance point is recognized the commotion will be lessened and hand extraction will be finished. Presently to discover the state of 
hand, highlight acknowledgment is completed for facilitate grouping stage. In the wake of perceiving hand represents those hand signals will be use in mouse free interface wherein left snap, right snap capacity will be incorporated. Utilizing of this mouse free interface there will simplicity of utilizing mouse work by motions. [6]

Motions are parts of discourse, not backups or 'additional items' (ADAM KENDON'S 2008 term), all things considered vital parts of it. Much proof backings this thought, however its full suggestions have not generally been perceived. The signals I mean are ordinary events - the unconstrained, unwitting, and standard backups of discourse that we find in our moving fingers, hands and arms. They are so much a piece of talking that one is frequently ignorant of them, however in the event that you glance around and watch somebody talking in casual terms you are probably going to see the hands and arms in movement. Motions of this kind resemble watching somebody's idea in movement. The development point, or GP, plans to explain this understanding (e.g., McNeill 1992, 2005). GPs are proposed dynamic units of dialect and thought. In a GP, synchronized signal symbolism and phonetic categorical substance shape an unbreakable thought unit. Discourse, being fixing to thought, is as needs be inalienably multimodal - both vocal-etymological and manual-gestural - and the subsequent semiotic restriction (a similar thought in two inverse semiotic modes in the meantime) powers micro genetic change (the GP, looking for conclusion, is "unloaded" into a static, i.e., a maximally steady, syntactic shape: practically, finding a thoughtfully perfect development/semantic casing is the symbolism dialect logic's 'stop-arrange'). [7]

Motion (Gesture) implies a development of part of body. Motion Recognition is the innovation that perceives the development of body. It perceives hand, arms, head or any piece of the body. So the objective of Gesture Recognition is to give interface of human body with PC through numerical calculation. This paper gives a continuous vision based strategy for perceiving human body movement utilizing MATLAB. It additionally gives the working points of interest of perceiving process utilizing Edge recognition and Skin identification calculations. In this paper Gesture Recognition used to control the media application over LAN availability and remote equipment interface innovation utilized enable us to control home machines shaped in a remote system through Hand motion development just, this opens another pleothera of roads progressively vision based home robotization framework. Additionally detail inquire about is centered around giving security mechanism through motion. We utilize body movement for validation reason or give security to get to the data or information with CCTV camera. It will be a multipurpose framework. Perceive an example of body movement with CCTV camera and used to give security to framework not additional webcam is utilized. [8]

With a specific end goal to construe aim from signal, a simple classification of sorts of motions into five primary classes is presented. The classification is planned as a reason for fusing the comprehension of motion into human-robot collaboration (HRI). A few prerequisites for the operational classification of motion by a robot collaborating with people are likewise proposed. The classification introduced here recommends a few prerequisites for the plan and execution of frameworks deriving aim from motion in view of this classification. These necessities may be acknowledged in a assortment of various ways utilizing, e.g. persistent low-key following or more point by point examination, occasion based or potentially situation based acknowledgment, and expectation of human movement in light of models of human action lows (with or without acknowledgment of specific people what's more, their past connections), depending the specific needs of the given human-robot cooperation plan and the requirements and specificity of its expected operational setting. Outline of a robot confined to aiding dependably a similar client in the kitchen condition would be very not quite the same as one that ought to be a more broadly useful hireling or buddy in a home domain containing a few grown-ups, youngsters and pets, yet the classification introduced here is pertinent in educating the plan of motion acknowledgment for inducing plan in eithersort of framework, and for outlining other HRI frameworks. [9]

\section{METHODOLOGY}

\section{FRAMEWORK MODEL}

Information: The contribution of the motion acknowledgment is hand motion picture that taken by a webcam or camera. This is a movement of hand or body part that is caught and prepared by edge location calculation. Before preprocessing first we instate variable and parameters.

Preprocessing: Preprocessing is connected to pictures before we can separate components from hand pictures. It is a well ordered procedure to perceive and coordinating appearance of the picture that is already put away.

STEP 1: Captured picture is a dark scale picture changed over into break even with twofold shape or edges. Ostu calculation is utilized to change over dark scale into paired frame. In PC vision and picture handling, Otsu's technique is utilized to naturally perform histogram shape-based picture thresholding or the lessening of a dark level picture to a parallel picture. The calculation accept that the picture to be limit contains two classes of pixels or bi-modular histogram (e.g. closer view and foundation) at that point computes the ideal edge isolating those two classes with the goal that their joined spread (intraclass change) is negligible. The consequence of this progression is in the figure appear underneath.

STEP 2: After applying the Otsu calculation on the first dark scale picture, we locate that some commotion happens in paired picture or casings. These mistakes make issues in location of hand motion. So we require expel these mistakes. Morphological separating approach is done to expel these mistakes. In the morphological sifting, we apply a control on the twofold picture. The estimation of any given pixel in the yield picture is acquired by associating set of tenets on the neighbors in the information picture. STEP 3: After finding the genuine double picture highlights extraction is perform. Components extraction is a procedure of discover the edge of recognized picture. Watchful edge identification calculation is utilized for discover the edge of recognized picture.

STEP4: The distinguished edge of picture is utilized to coordinate the presence of hand signal and give the outcome to the yield equipment or media application. $[5,6,7,8,9,10]$ 


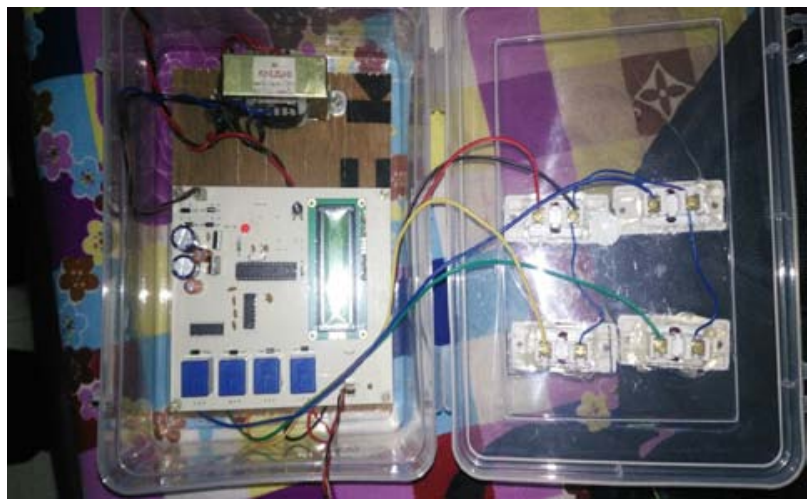

Fig 2: Hardware Implementation

\section{RESULT}

When complete the project we apply all the things for result and then we get all the result is prefect according to my project direction. We are set the four hand action for operate the function that are show in figure.

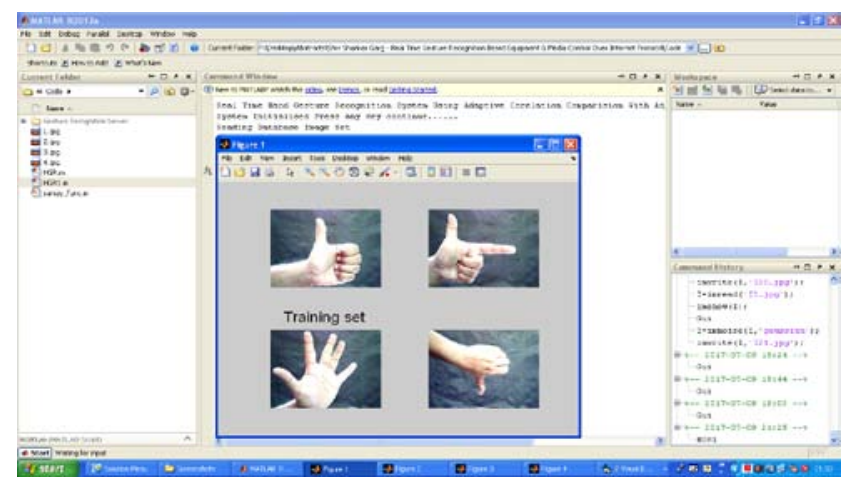

Fig 3: All set image for operations

Action and its work.

Image 1: Play The Song.

Image 2: Pause and Play the Song

Image 3: Open a Notebook

Image 4: Open a Image

\section{Action 1}

When we apply first image then system capture and perform the set operation.

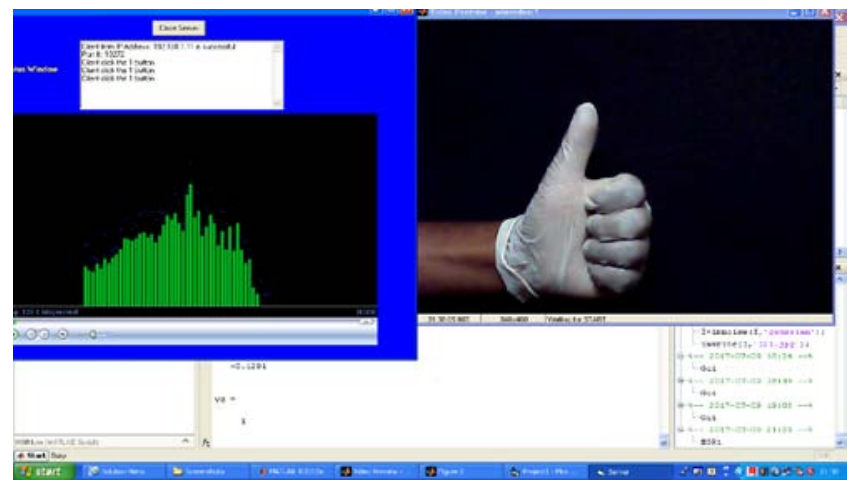

Fig 4: Action 1 for play the song

\section{Action 2}

When we apply second image then system capture and perform the set operation.

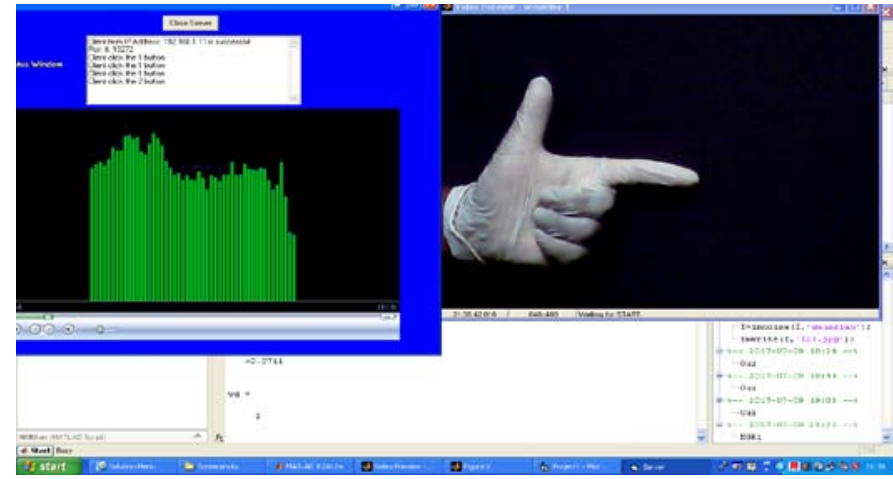

Fig 5: Action 2 for pause and play the song

\section{Action 3}

When we apply third image then system capture and perform the set operation.

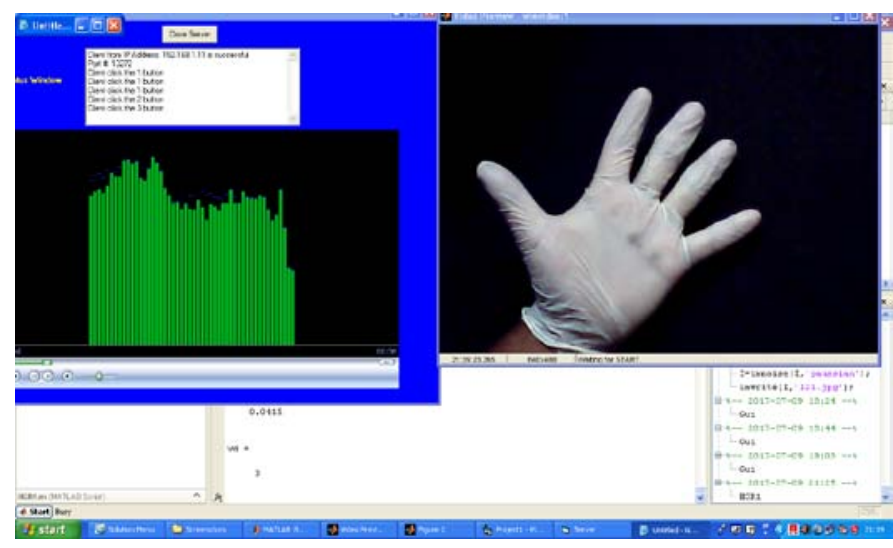

Fig 6:Action 3 for open a Notebook

\section{Action 4}

When we apply four image then system capture and perform the set operation.

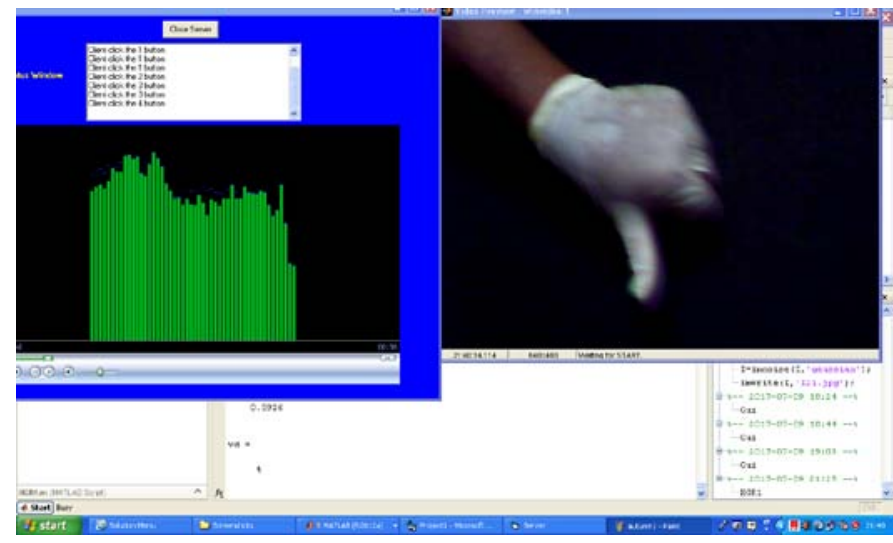

Fig 7: Action 4 for open an Image

\section{CONCLUSION AND FUTURE SCOPE}

A PC vision calculation is recommended that perceives the four hand signals in particular: Play, Stop, Forward, and Reverse, for controlling media player utilizing neural system. An edge from the webcam camera was caught, at that point 
skin division utilizing LAB shading space was utilized to fragment skin locales from foundation pixels. Another picture was made containing hand limit of the client's hand. A curved structure also, corner location are utilized to portray the shape elements of hand motion. A regulated back-spread multi-layer feedforward neural system was utilized for arrangement of client's hand motions too. The order was made, without the requirement for utilizing any exceptional devices, for example, gloves or marker. Be that as it may, the proposed calculation performed well in order the four clients' hand signal summon, with some order mistakes. The normal grouping rate of $95 \%$ was gotten for the testing sets. As future work, we plan to utilize hand motion grouping calculation for controlling the $\mathrm{TV}$, and portable applications.

\section{REFERENCES}

[1] C. L. NEHANIV. K, DAUTENHAHN. J, KUBACKI. M, HAEGELE, M., PARLITZ, C. \& ALAMI, R. A methodological approach relating the classification of gesture to identification of human intent in the context of human-robot interaction. Robot and Human Interactive Communication, 2005. IEEE, 371-377.

[2] A. Billard, Y. Epars, S. Calinon, G. Cheng, and S. Schaal. Discovering optimal imitation strategies. Robotics \&
Autonomous Systems, Special Issue: Robot Learning from Demonstration, 47(2-3): 69.77, 2004.

[3] A. Bonnaccorsi, "On the Relationship between Firm Size and Export Intensity” Journal of International Business Studies, XXIII (4), pp. 605-635, 1992. (journal style)

[4] David McNeill "Gestures of Power and the Power of Gestures" Proceedings of the Berlin Ritual-Conference 2008.

[5] Hamid A. Jalab, Herman .K. Omer "Human Computer Interface Using Hand Gesture Recognition Based On Neural Network" IEEE 2015.

[6] Kashmera Khedkkar Safaya, Prof.(DR.). J.W.Bakal "Real Time Based Bare Hand Gesture Recognition” IIJIT 2013.

[7] AUERBACH, E. 2003. Mimesis: The Representation of Reality in Western Literature. Princeton, N.J.: Princeton University Press.

[8] Chrystopher L. Nehaniv “Classifying Types of Gesture and Inferring Intent” AISB 2005.

[9] Tarachand Saini,Savita Sivani "Real Time Vision Hand Gesture Recognition Based Media Control via LAN \& Wireless Hardware Control” IJMER 2013.

[10] P. Jenifer Martina1, P. Nagarajan2, P. Karthikeyan3, "Hand Gesture Recognition Based Real-time Command System” 1P.G Scholar/Applied Electronics, Dept of ECE, PSNA College of Engineering and Technology Dindigul, Tamilnadu, India,April 2013. 